\title{
Correction to: Sex and race differences of cerebrospinal fluid metabolites in healthy individuals
}

\author{
Zackery W. Reavis ${ }^{1,6} \cdot$ Nikhil Mirjankar $^{2}$. Srikant Sarangi ${ }^{2} \cdot$ Stephen H. Boyle ${ }^{3}$ Cynthia M. Kuhn ${ }^{1}$ Wayne R. Matson ${ }^{2}$. \\ Michael A. Babyak ${ }^{3}$. Samantha A. Matson ${ }^{2} \cdot$ Ilene C. Siegler $^{3} \cdot$ Rima Kaddurah-Daouk $^{3} \cdot$ Edward C. Suarez $^{3}$. \\ Redford B. Williams ${ }^{3} \cdot$ Katherine Grichnik ${ }^{4} \cdot$ Mark Stafford-Smith $^{5} \cdot$ Anastasia Georgiades $^{7}$ (1)
}

Received: 1 June 2021 / Accepted: 1 June 2021 / Published online: 9 June 2021

○) Springer Science+Business Media, LLC, part of Springer Nature 2021

\section{Correction to: Metabolomics (2021) 17:13 https://doi.org/10.1007/s11306-020-01757-0}

After publication of our article (Reavis et al., 2021) it came to our attention that the values for CSF melatonin levels in Tables 3 and 4 and in the histogram depicting melatonin levels in Supplemental Online Resource 2 seemed abnormally high. The CSF levels of the metabolites were quantitated by a high-performance liquid chromatography coupled with electrochemical coulometric array detection (LCECA).

Because the CSF melatonin levels were expected to be very low, they were determined using a separate method. The chromatographic data were exported as "digital maps" which allow somewhat greater sensitivity in detecting low concentration values than the use of our peak finding algorithm.

Values from the digital maps are typically initially exported as a $\%$ of the pool and appended to the peak tables

The original article can be found online at https://doi.org/10.1007/ s11306-020-01757-0.

Anastasia Georgiades

anastasia.georgiades@duke.edu

1 Department of Pharmacology \& Cancer Biology, School of Medicine, Duke University, Durham, NC, USA

2 Ixcela, The Internal Fitness ${ }^{\mathrm{TM}}$ Company, Bedford, MA, USA

3 Department of Psychiatry \& Behavioral Sciences, School of Medicine, Duke University, Durham, NC, USA

4 American Anesthesiology, Sunrise, FL, USA

5 Department of Anesthesiology, School of Medicine, Duke University, Durham, NC, USA

6 College of Medicine, University of Central Florida, Orlando, FL, USA

7 Department of Psychiatry \& Behavioral Sciences, School of Medicine, Duke University, Box 3454 DUMC, Durham, NC 27710, USA to make them compatible with the values in $\%$ of pool from standard peak finding algorithms. The actual concentrations for the compounds measured by digital maps in the pool are determined with a separate set of low-level standards. In the case of this study, melatonin concentrations were exported as a $\%$ of the pool with a $10 \mathrm{pg} / \mathrm{mL}$ standard and these values were used to append the data table from the peak finding algorithm, which is based on a standard of $\mathrm{ng} / \mathrm{mL}$. Thus, conversion of $\%$ of pool to concentration resulted in the 100 fold error in melatonin values in the tables of our manuscript because they were presented in units of $\mathrm{pg} / \mathrm{mL}$ instead of $\mathrm{ng} /$ $\mathrm{mL}$. This has now been corrected by dividing the values for melatonin by 100 .

The corrected melatonin values are presented in Tables 3 and 4 as well as in the histogram provided below. This error does not impact the results presented for melatonin or the conclusions of the article. This error does not affect any of the other metabolomics variables. The authors apologize for this mistake and want to thank Dr. David Kennaway for noticing the inaccuracy.

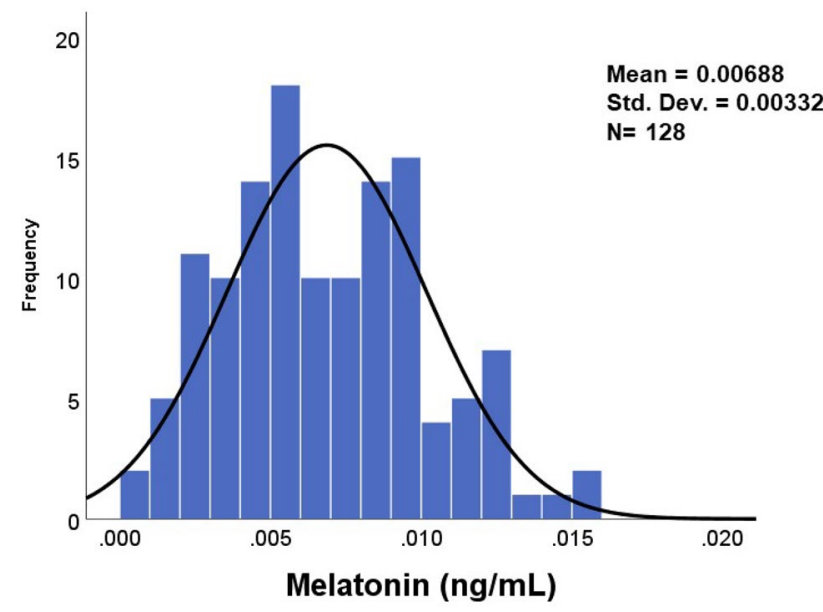


Table 3 Sex differences in CSF metabolite levels (ng/mL) and ratios (medians and 25th and 75th quartiles)

\begin{tabular}{|c|c|c|c|c|}
\hline & $\begin{array}{l}\text { Women } \\
\text { Median (25th-75th) }\end{array}$ & $\begin{array}{l}\text { Men } \\
\text { Median (25th-75th) }\end{array}$ & p-value & $\mathrm{N}$ \\
\hline \multicolumn{5}{|l|}{ Metabolite } \\
\hline 2-Hydroxyphenylacetic acid & $4.77(4.21-5.30)$ & $4.98(4.52-5.62)$ & 0.195 & 129 \\
\hline 3-Hydroxykynurenine & $1.03(0.51-2.82)$ & $0.94(0.41-3.31)$ & 0.909 & 129 \\
\hline 3-O-methyldopa & $1.09(0.81-1.42)$ & $1.28(0.68-2.21)$ & 0.386 & 105 \\
\hline 4-Hydroxyphenyllactic acid & $43.19(35.75-58.08)$ & $43.32(32.49-56.26)$ & 0.670 & 129 \\
\hline 5-HIAA & $13.60(10.61-19.63)$ & $10.26(6.33-14.21)$ & 0.0011 & 129 \\
\hline 5-HTP & $1.13(0.84-1.39)$ & $1.07(0.79-1.33)$ & 0.358 & 129 \\
\hline 7-Methylxanthine & $2.06(1.65-2.57)$ & $2.17(1.67-2.46)$ & 0.585 & 129 \\
\hline Alpha-Tocopherol & $120.82(100.49-141.71)$ & $126.33(95.64-152.58)$ & 0.775 & 129 \\
\hline Anthranilic acid & $2.09(1.66-2.36)$ & $2.16(1.70-2.36)$ & 0.563 & 123 \\
\hline Ascorbate & $24,127.02(20,230.46-30,557.56)$ & $23,496.17(17,300.34-31,353.01)$ & 0.515 & 129 \\
\hline Cysteine & $6.64(5.36-8.71)$ & $10.5(7.90-13.62)$ & $<.0001$ & 129 \\
\hline DOPAC & $1.17(0.70-2.03)$ & $1.06(0.58-1.49)$ & 0.095 & 117 \\
\hline Guanosine & $3.62(3.06-4.32)$ & $3.40(2.98-4.01)$ & 0.302 & 121 \\
\hline Glutathione & $12.28(10.81-13.34)$ & $14.34(9.88-15.69)$ & 0.062 & 90 \\
\hline Glutathione disulfide & $10.52(8.20-13.22)$ & $9.95(7.91-13.84)$ & 0.797 & 129 \\
\hline Gamma-tocopherol & $22.63(12.37-31.49)$ & $24.56(15.44-37.10)$ & 0.208 & 125 \\
\hline HVA & $37.76(26.09-54.75)$ & $34.06(24.15-45.05)$ & 0.107 & 129 \\
\hline Hypoxanthine & $722.26(550.38-909.68)$ & $635.42(508.92-815.96)$ & 0.178 & 129 \\
\hline Indoleacetic acid & $3.47(2.43-5.67)$ & $2.76(2.15-5.08)$ & 0.119 & 126 \\
\hline Indolelactic acid & $1.86(1.35-2.42)$ & $1.91(1.45-2.62)$ & 0.369 & 111 \\
\hline Kynurenine & $5.07(3.39-6.30)$ & $4.83(3.33-6.90)$ & 0.910 & 129 \\
\hline L-Dopa & $0.95(0.71-1.35)$ & $0.94(0.69-1.21)$ & 0.473 & 127 \\
\hline Melatonin & $0.0067(0.0042-0.0093)$ & $0.0065(0.0042-0.0092)$ & 0.892 & 128 \\
\hline Methionine & $955.53(796.19-1125.83)$ & $1033.47(849.31-1251.00)$ & 0.118 & 129 \\
\hline MHPG & $10.39(8.88-11.76)$ & $10.48(9.46-11.55)$ & 0.446 & 129 \\
\hline $\mathrm{N}$-acetylserotonin & $1.04(0.69-1.27)$ & $1.14(0.88-1.55)$ & 0.049 & 129 \\
\hline Tryptophol & $11.67(8.37-15.52)$ & $13.10(9.67-17.92)$ & 0.176 & 129 \\
\hline Tryptophan & $485.29(417.20-550.93)$ & $493.52(442.82-566.98)$ & 0.348 & 129 \\
\hline Tyrosine & $2033.30(1660.71-2283.83)$ & $2023.74(1744.40-2372.01)$ & 0.298 & 129 \\
\hline Uric acid & $5353.71(4041.17-7102.65)$ & $9008.48(7033.92-11,906.72)$ & $<.0001$ & 129 \\
\hline Vanillylmandelic acid & $0.76(0.58-1.00)$ & $0.71(0.43-1.05)$ & 0.268 & 128 \\
\hline Xanthine & $324.28(276.36-392.87)$ & $320.78(276.85-377.55)$ & 0.870 & 129 \\
\hline Xanthosine & $23.74(20.25-28.26)$ & $23.10(19.41-28.82)$ & 0.844 & 129 \\
\hline \multicolumn{5}{|l|}{ Ratios } \\
\hline 5-HTP/Trp & $0.0024(0.0018-0.0029)$ & $0.0021(0.0016-0.0027)$ & 0.147 & 129 \\
\hline 5-HIAA/5-HTP & $12.37(8.47-19.99)$ & $9.49(6.20-15.96)$ & 0.030 & 129 \\
\hline Uric/xanthine & $16.34(11.77-19.58)$ & $30.20(21.61-36.93)$ & $<.0001$ & 129 \\
\hline Xanthine/hypoxanthine & $0.47(0.40-0.57)$ & $0.51(0.41-0.64)$ & 0.215 & 129 \\
\hline
\end{tabular}


Table 4 Race differences in CSF metabolite levels $(\mathrm{ng} / \mathrm{mL})$ and ratios (medians and 25th and 75th quartiles

\begin{tabular}{|c|c|c|c|c|}
\hline & $\begin{array}{l}\text { African American } \\
\text { Median (25th-75th) }\end{array}$ & $\begin{array}{l}\text { White } \\
\text { Median (25th-75th) }\end{array}$ & p-value & $\mathrm{N}$ \\
\hline \multicolumn{5}{|l|}{ Metabolite } \\
\hline 2-Hydroxyphenylacetic acid & $4.94(4.41-5.48)$ & $4.84(4.30-5.26)$ & 0.643 & 129 \\
\hline 3-Hydroxykynurenine & $1.80(0.49-3.51)$ & $0.72(0.50-1.37)$ & 0.018 & 129 \\
\hline 3-O-methyldopa & $1.22(0.82-1.88)$ & $1.04(0.69-1.45)$ & 0.069 & 105 \\
\hline 4-Hydroxyphenyllactic acid & $43.32(31.25-57.56)$ & $43.97(36.09-55.90)$ & 0.543 & 129 \\
\hline 5-HIAA & $12.33(8.99-17.95)$ & $11.76(8.41-16.00)$ & 0.334 & 129 \\
\hline 5-HTP & $1.01(0.79-1.36)$ & $1.16(0.85-1.34)$ & 0.498 & 129 \\
\hline 7-Methylxanthine & $1.98(1.61-2.38)$ & $2.20(1.84-2.80)$ & 0.037 & 129 \\
\hline Alpha-Tocopherol & $116.99(94.60-137.53)$ & $132.56(111.66-151.31)$ & 0.007 & 129 \\
\hline Anthranilic acid & $2.13(1.59-2.38)$ & $2.12(1.71-2.32)$ & 0.907 & 123 \\
\hline Ascorbate & $25,197.53(19,613.42-30,802.89)$ & $23,585.38(15,970.69-30,411.53)$ & 0.354 & 129 \\
\hline Cysteine & $7.87(5.60-9.57)$ & $9.01(6.67-12.91)$ & 0.029 & 129 \\
\hline DOPAC & $1.18(0.74-1.91)$ & $0.99(0.59-1.45)$ & 0.110 & 117 \\
\hline Guanosine & $3.34(2.96-4.18)$ & $3.60(3.16-4.27)$ & 0.099 & 121 \\
\hline Glutathione & $12.64(9.75-15.63)$ & $12.88(11.69-14.89)$ & 0.768 & 90 \\
\hline Glutathione Disulfide & $9.60(8.03-13.24)$ & $11.19(8.40-13.72)$ & 0.166 & 129 \\
\hline Gamma-Tocopherol & $21.19(14.84-33.92)$ & $25.20(12.24-35.91)$ & 0.519 & 125 \\
\hline HVA & $36.54(25.94-54.58)$ & $34.41(25.21-45.63)$ & 0.503 & 129 \\
\hline Hypoxanthine & $669.10(530.06-835.36)$ & 640.87 (513.24-911.57) & 1.000 & 129 \\
\hline Indoleacetic acid & $2.63(2.01-4.08)$ & $4.83(2.76-6.41)$ & $<.0001$ & 126 \\
\hline Indolelactic acid & $1.87(1.35-2.43)$ & $1.91(1.58-2.52)$ & 0.406 & 111 \\
\hline Kynurenine & $4.30(3.13-5.92)$ & $5.83(4.84-7.31)$ & $<.0001$ & 129 \\
\hline L-Dopa & $0.95(0.70-1.26)$ & $0.94(0.71-1.27)$ & 0.861 & 127 \\
\hline Melatonin & $0.0059(0.0039-0.0083)$ & $0.0080(0.0049-0.0098)$ & 0.036 & 128 \\
\hline Methionine & $974.01(806.38-1221.82)$ & $982.47(843.03-1169.90)$ & 0.909 & 129 \\
\hline MHPG & $10.58(9.32-11.55)$ & $9.95(8.44-11.70)$ & 0.182 & 129 \\
\hline $\mathrm{N}$-acetylserotonin & $1.06(0.85-1.33)$ & $1.10(0.78-1.49)$ & 0.610 & 129 \\
\hline Tryptophol & $11.56(8.03-16.43)$ & $12.76(10.34-17.61)$ & 0.111 & 129 \\
\hline Tryptophan & 484.05 (419.09-565.47) & $491.69(435.12-556.50)$ & 0.531 & 129 \\
\hline Tyrosine & $2025.29(1696.14-2347.68)$ & $2008.01(1686.39-2363.03)$ & 0.883 & 129 \\
\hline Uric acid & $6749.83(4619.79-8800.71)$ & $7572.44(5374.56-11,723.86)$ & 0.081 & 129 \\
\hline Vanillylmandelic acid & $0.75(0.46-1.05)$ & $0.74(0.52-0.98)$ & 0.953 & 128 \\
\hline Xanthine & $311.72(266.49-355.13)$ & $354.36(305.15-412.95)$ & 0.0012 & 129 \\
\hline Xanthosine & $23.97(20.59-29.34)$ & $23.24(17.83-25.84)$ & 0.062 & 129 \\
\hline \multicolumn{5}{|l|}{ Ratios } \\
\hline Indoleacetic acid/tryptophan & $0.0057(0.0041-0.0086)$ & $0.0090(0.0056-0.0128)$ & 0.0001 & 126 \\
\hline Anthranilic acid/indoleacetic acid & $0.69(0.45-1.03)$ & $0.43(0.29-0.70)$ & 0.0002 & 121 \\
\hline Kynurenine/tryptophan & $0.0088(0.0065-0.0125)$ & $0.0119(0.0094-0.0149)$ & 0.0002 & 129 \\
\hline Anthranilic acid/kynurenine & $0.48(0.33-0.68)$ & $0.34(0.25-0.45)$ & 0.0002 & 123 \\
\hline 3-Hydroxykynurenine/Kynurenine & $0.48(0.11-0.98)$ & $0.12(0.07-0.18)$ & 0.0004 & 129 \\
\hline Uric/xanthine & $20.89(14.48-30.61)$ & $20.05(14.84-33.76)$ & 0.615 & 129 \\
\hline Xanthine/hypoxanthine & $0.47(0.40-0.58)$ & $0.53(0.46-0.66)$ & 0.016 & 129 \\
\hline
\end{tabular}

\section{Reference}

Reavis, Z. W., Mirjankar, N., Sarangi, S., Boyle, S. H., Kuhn, C. M., Matson, W. R., Babyak, M. A., Matson, S. A., Siegler, I. C., Kaddurah-Daouk, R., Suarez, E. C., Williams, R. B., Grichnik, K., Stafford-Smith, M., \& Georgiades, A. (2021). Sex and race differences of cerebrospinal fluid metabolites in healthy individuals. Metabolomics, 17(2), 13. https://doi.org/10.1007/ s11306-020-01757-0

Publisher's Note Springer Nature remains neutral with regard to jurisdictional claims in published maps and institutional affiliations. 